\title{
Good ACE, bad ACE do battle in lung injury, SARS
}

\author{
John Nicholls \& Malik Peiris
}

\begin{abstract}
Two studies show how the enzyme ACE2 protects against lung injury caused by SARS and other agents. ACE2 seems to counteract the effects of ACE, which are more damaging (pages 875-879).
\end{abstract}

In 1918, as World War I came to a close, clinicians and pathologists tried to come to grips with an unusual new pneumonic illness. In the lungs of the individuals who died of this illness they found "homogeneous structureless non-cellular exudates which fills the bronchioles and...forms as it were a plastering round the inside of the alveoli... and is very similar to that which can be seen in fatal cases of poison-

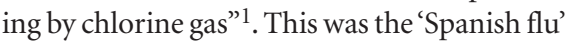
influenza pandemic, which is estimated to have killed over 40 million people worldwide.

This pattern of injury is now called diffuse alveolar damage (DAD), and is the histological change associated with acute respiratory distress syndrome (ARDS). DAD is a common reaction to pneumocyte damage and may be initiated by noxious gases and infective agents, including severe acute respiratory syndrome coronavirus (SARS-CoV) and influenza ${ }^{2}$. DAD has a high mortality rate and, other than supportive clinical care, there are few specific therapeutic options of proven benefit.

In a recent issue of Nature, Imai et al. ${ }^{3}$ examine $\mathrm{DAD}$ in a mouse model, and highlight the role of the renin-angiotensin system, an endocrine cascade best known for regulating blood pressure in DAD. The authors show that an enzyme in this cascade, angiotensin-converting enzyme 2 (ACE2), protects against lung damage. In this issue of Nature Medicine, researchers from the same group also find that ACE2 is downregulated in mice infected with SARS-CoV, contributing to lung injury in this disease 4 .

The functional unit of the lung is the alveolus, lined mainly by two types of cells-large, flattened, nonreplicating type 1 pneumo-

John Nicholls is in the Department of Pathology,

The University of Hong Kong and Malik Peiris in the Department of Microbiology, The University of Hong Kong, Hong Kong, SAR.

e-mail: nicholls@pathology.hku.hk

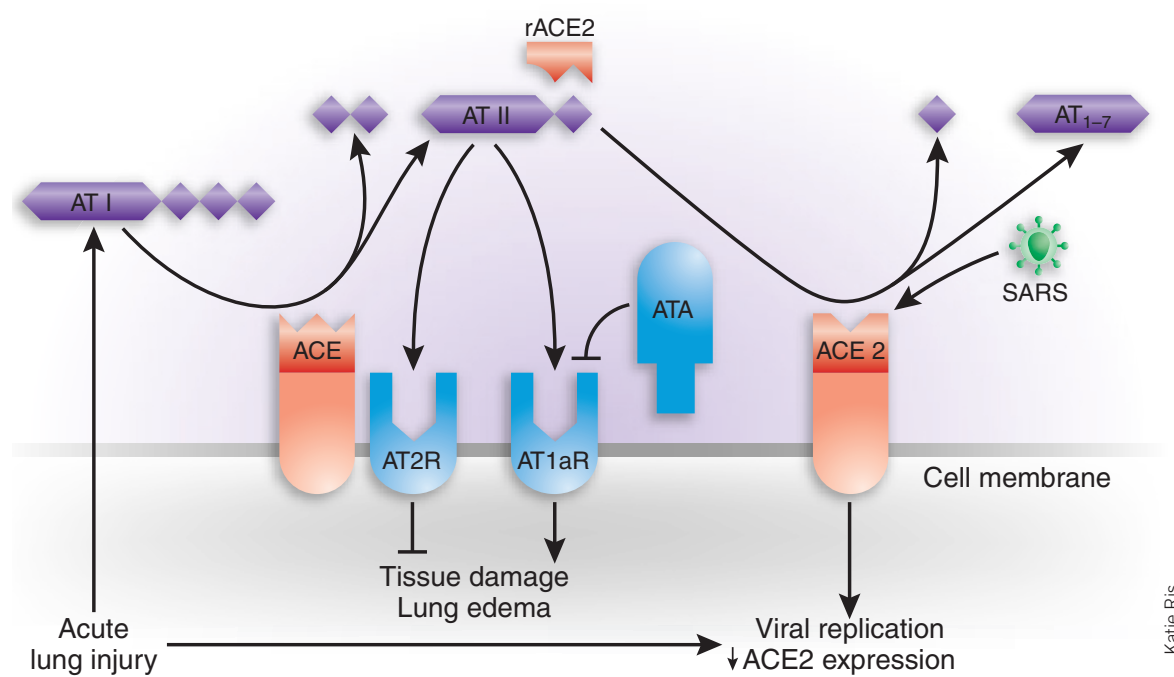

Figure 1 Simplified schematic representation of angiotensin-converting enzyme (ACE) regulation of acute lung injury. ACE converts angiotensin I (AT I) to angiotensin II (AT II) which binds to either angiotensin II receptor 1a (AT1aR), leading to tissue damage and lung edema, or to angiotensin II receptor 2 (AT2R), reducing tissue damage. Angiotensin-converting enzyme 2 (ACE2) in turn converts the potent AT II to a less damaging angiotensin ${ }_{1-7}\left(A T_{1-7}\right)$. SARS binds to ACE2, resulting in downregulation through its internalization, and thus reduced inactivation of AT II. Lipopolysaccharide, sepsis and acid treatment also results in ACE2 downregulation. Administration of recombinant ACE2 (rACE2) reduces lung damage by inactivation of AT II and treatment with AT 1aR antagonists (ATA) may also have the potential to reduce lung damage.

cytes involved in gas exchange and smaller type 2 pneumocytes. The latter produce surfactant and can differentiate into type 1 pneumocytes when the need arises, such as when type 1 pneumocytes are damaged. In $\mathrm{DAD}$, pneumocyte destruction leads to a disruption of the alveolar-vessel interface and a consequent outpouring of serum proteins that form the hyaline membrane, identified in 1919 as the "non-cellular exudate" 1 .

Work in cell lines has suggested that ACE2 is the functional receptor for SARS-CoV ${ }^{5,6}$. Kuba et al. ${ }^{4}$ provide the first evidence in vivo of the importance of ACE2 for SARS-CoV replication in mice.
Angiotensin-converting enzyme (ACE) cleaves two amino acids from the prohormone angiotensin I to produce the octapeptide angiotensin II-a potent vasoconstrictor and stimulator of cardiac growth (Fig. 1). Whereas ACE was discovered more than 50 years ago, it was only in 2000 that ACE2 was characterized. Although similar in some ways to ACE, ACE2 functions by removing a single phenylalanine from the amino acid chain, converting angiotensin II to angiotensin ${ }_{1-7}$. Angiotensin 1 ${ }_{7}$ has opposing functions to angiotensin II, acting as a potent vasodilator and repressor of cardiac growth ${ }^{7}$. ACE2 has also been found to hydrolyze peptides outside of the renin- 
antiotensin system, including dynorphin A (1-13), apelin-13 and des-Arg(9) bradykinin, but the exact functional role of ACE2 in these peptide systems has yet to be revealed.

There is now an increasing body of evidence supporting the role of an activated reninangiotensin system in acute lung injury. ACE levels increase in the bronchoalveolar fluid of individuals with $\mathrm{ARDS}^{8}$, and ACE is thought to influence both vascular permeability and the air-vessel interface, as well as maintain pneumocyte viability. Furthermore, treatment of rats with acute lung injury using ACE antagonists delays the onset of ARDS 9 .

But what of the role of ACE2 in acute lung injury? Imai et al. ${ }^{3}$ show that ACE2 has an opposing function to ACE and protects against lung injury. The authors found that systemic treatment with recombinant ACE2 reduced lung injury, suggesting that such an approach might be able to treat DAD.

The protective effect of ACE2 seems to result partially from the conversion of angiotensin II by ACE2 to angiotensin ${ }_{1-7}$, thereby reducing angiotensin II binding to the cell membrane receptors AT1aR (angiotensin II type 1a receptor) and AT2R (angiotensin II type 2 receptor). It is believed that angiotensin II binding to AT1aR will stimulate lung injury, whereas binding to AT2R reduces lung injury. Kuba et al. ${ }^{4}$ propose that binding of SARS-CoV to ACE2 downregulates ACE2, thus leaving angiotensin II unmodified, allowing it to continue to bind to the AT1aR to aggravate the lung injury and produce lung edema (Fig. 1). Whether other ACE2 products and their receptors are involved in SARS remains to be determined.

Despite these insights into the pathogenesis of SARS and DAD, a number of questions arise. Conflicting results obtained by northern blotting, RT-PCR and immunohistochemistry of ACE2 in the human lung leave the organ and cell expression of ACE2 unresolved ${ }^{10,11}$. Furthermore, SARS-CoV infection in mice does not produce the typical DAD picture seen in human disease $\mathrm{e}^{12}$.

There also are differences in the receptor binding affinity of SARS-CoV-like viruses with human ACE2, compared with mouse ACE2. The Spike proteins of the humanadapted SARS-CoV responsible for the 2003 global outbreak of SARS bound more efficiently to human ACE2 than the Spike protein of the animal precursor virus-thus explaining the apparently milder nature of the SARS-like infections caused by the animal precursor virus in December 2003-January 2004. Furthermore, the newly discovered coronavirus NL63 also binds to ACE2 ${ }^{13}$ — but unlike SARS-CoV, the NL63 coronavirus is a ubiquitous human pathogen and is not generally associated with ARDS or DAD. The use of ACE2 as a viral receptor of itself therefore does not invariably result in aggravating acute lung injury, leading to DAD. In this regard, a detailed comparison between NL63 and SARS$\mathrm{CoV}$ is called for.

Clearly, the potential therapeutic utility of recombinant ACE2 and angiotensin II receptor inhibitors-already in clinical use for control of blood pressure-for acute lung injury resulting from viruses and other causes will be a productive field for investigation. This is particularly relevant as we prepare to confront a potential avian flu pandemic, armed with only a limited number of therapeutic options.

1. Abrahams, A., Hallows, N. \& French, H. Lancet 196 1-11 (1919).

2. Nicholls, J.M. et al. Lancet 361, 1773-1778 (2003)

3. Imai, Y. et al. Nature 436, 112-116 (2005).

4. Kuba, K. et al. Nat. Med. 11, 875-879 (2005).

5. Li, W. et al. Nature 426, 450-454 (2003)

6. Xiao, X., Chakraborti, S., Dimitrov, A.S., Gramatikoff, K. \& Dimitrov, D.S. Biochem. Biophys. Res. Commun. 312, 1159-1164 (2003).

7. Turner, A.J., Hiscox, J.A. \& Hooper, N.M. Trends Pharmacol. Sci. 25, 291-294 (2004).

8. Idell, S. et al. Chest 91, 52-56 (1987).

9. Raiden, S. et al. J. Pharmacol. Exp. Ther. 303, 45-51 (2002).

10. Harmer, D., Gilbert, M., Borman, R. \& Clark, K.L. FEBS Lett. 532, 107-110 (2002).

11. Hamming, I. et al. J. Pathol. 203, 631-637 (2004).

12. Roberts, A. et al. J. Virol. 79, 5833-5838 (2005).

13. Hofmann, H. et al. Proc. Natl. Acad. Sci. USA 102 7988-7993 (2005).

\title{
TZDs and diabetes: testing the waters
}

\section{Clay F Semenkovich}

\author{
The use of thiazolidinediones, drugs for type 2 diabetes that may also be useful for other chronic inflammatory \\ disorders, is limited by fluid retention. Research in mice identifies a mechanism for this side effect and introduces a \\ potential remedy (pages $861-866$ ).
}

Many individuals with type 2 diabetes find that taking their medications introduces new medical problems into their lives. Thiazolidinediones (TZDs) enhance insulin sensitivity, lower blood glucose and suppress inflammation-but they can also cause life-threatening fluid retention and edema. This side effect limits the potential of these drugs for other inflammatory conditions such as atherosclerosis.

The author is in the Division of Endocrinology, Metabolism, and Lipid Research, Washington University School of Medicine, St. Louis, Missouri 63110, USA.

e-mail: csemenko@wustl.edu
Two new studies, published in the Proceedings of the National Academy of Sciences $^{1}$ and in this issue of Nature Medicine ${ }^{2}$, show how TZDs cause edema by altering the handling of salt balance in the renal collecting duct, a structure where water is extracted from the urine. TZDs cause increased salt uptake in the duct through activation of their target, the multitasking nuclear receptor PPAR $\gamma$.

PPAR $\gamma$ is a member of a subfamily of nuclear hormone receptors, the PPAR proteins. These proteins are activated by fatty acids, and may be involved in a host of disorders characterized by abnormal fatty acid metabolism - including diabetes, the metabolic syndrome, atherosclerosis, chronic inflammatory states like asthma, infections such as HIV, and cancer. Binding of fatty acids causes PPARs to form heterodimers with the retinoid $\mathrm{X}$ receptor and induce gene expression in a ligand-dependent manner.

Two of the three proteins in the PPAR family are the targets of commonly used drugs. PPAR $\alpha$, predominantly in liver and muscle, is activated by fibrate drugs like gemfibrozil that lower triglycerides and elevate HDL, or 'good,' cholesterol ${ }^{3}$. PPAR $\gamma$ is present at high levels in fat, with lower levels in blood vessels, kidney, liver and muscle. PPAR $\gamma$ is activated by TZDs ${ }^{4}$, which enhance insulin sensitivity, lower blood glucose and suppress inflammation. Exactly how TZDs exert all these effects is under intense study. 